\title{
LEY DE VERNER Y LENGUAS ROMANCES
}

\author{
Enrique Guiter
}

Cuando, en el año 1822, Jakob Grimm (1791-1867) publicó la segunda edición de su gramática alemana ${ }^{1}$, los lingüistas de entonces vieron en ella una verdadera revelación de la historia del consonantismo germánico.

Sin embargo, las correspondencias en las cuales se apoyaba Grimm, ya habían sido evidenciadas, desde hacía cuatro años ${ }^{2}$, por el dinamarqués Rasmus K. Rask (1787-1832). Pero la publicación de Grimm "in modo più perfetto veniva formulata..., per cui è generalmente nota col nome di Legge di Grim"3.

Si se comparan las consonantes iniciales de palabras inglesas y alemanas con las de sus correspondientes latinas, tomadas como base de referencia, se observa una especie de permutación circular, casi perfecta en el caso del inglés, prolongada por una segunda mutación, que sólo concierne las dentales y una de las labiales, en el caso del alemán. (Notemos que la letra $v$ tiene el mismo sonido que la letra $f$ en alemán).

$\begin{array}{llll} & \text { Latín } & \text { Inglés } & \text { Alemán } \\ \text { Labiales } & \text { pater } & \text { father } & \text { Vater } \\ & \text { breuis } & \text { preen } & \text { Pfriem } \\ & \text { frater (gr. fráter) } & \text { brother } & \text { Bruder } \\ \text { Dentales } & \text { tres } & \text { three } & \text { drei } \\ & \text { decem } & \text { ten } & \text { zehn } \\ & \text { foris (gr. thúra) } & \text { door } & \text { Tor }\end{array}$

1. J. Grimm - Deutsche Grammatik ( $2^{\mathrm{a}}$ ed.), Göttingen, 1822.

2. R.K. Rask - Undersögelse om det gamle Nordiske eller Islandske Sprogs Oprindelse. Köbenhavn, 1818.

3. C. Tagliavini - Grammatica comparata delle lingue germaniche. Bologna, 1961, 19. 


\section{ENRIQUE GUITER}

$\begin{array}{llll}\text { Velares } & \text { cornu } & \text { horn } & \text { Horn } \\ & \text { granu } & \text { corn } & \text { Korn } \\ & \text { habere } & \text { give } & \text { geben }\end{array}$

Decíamos que la permutación circular era "casi" perfecta, porque el latín manifiesta una flaqueza cuando presenta el fonema $f$ dos veces: una como labial y corresponde regularmente con una $b$ del inglés; pero otra como dental y corresponde entonces con una $d$ del inglés.

La comparación de los dos ejemplos del latín con el griego, nos enseña que el latín confundió th con $f$, quizá bajo la influencia del etrusco, que había favorecido particularmente el fonema $f$. (De un préstamo del griego pnéo "soplar", el etrusco proporcionó al latín un derivado fenestra, con un paso semántico análogo al del español viento/ventana).

Tomando en cuenta el doble papel de la $f$ latina, el suyo propio y el de substituto de $t$, la ley de Grimm funciona con una buena regularidad en las mutaciones de consonantes iniciales. "La notion de mutation apparaît ainsi comme une idée directrice féconde. Il n'est pas douteux que le mérite de l'avoir conçue revient à Jacob Grimm. Jusque là, il n'y avait qu'une collection de faits de correspondance, bien établis par des équations de vocabulaire. Grimm a le premier vu dans les deux mutations les traits typiques communs, et créé un concept" 4 .

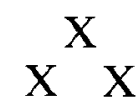

Sin embargo, la satisfacción de los lingüistas no resultó completa. Si la ley de Grimm funcionaba perfectamente con las consonantes iniciales, ofrecía irregularidades extrañas con las consonantes interiores.

Dos de los ejemplos presentados más arriba nos hacen percibir esa dificultad. La dental interior de la serie frater/brother/Bruder evoluciona exactamente como la dental inicial de la serie tres/three/drei; pero no pasa lo mismo con la dental interior de la serie pater/father/Vater.

En el inglés moderno hubo igualación gráfica de las consonantes interiores de brother y father; pero en el inglés antiguo el primer vocablo presentaba una th sorda, y el segundo una $d$ sonora. Luego a aquel nivel ya se había producido la anomalía: la segunda mutación consonántica del alemán trató la $d$ de fader exactamente como la de door.

La comparación del germánico, que siempre pone el acento en la sílaba inicial, y del latín, que no lo pone nunca en la sílaba final, no podía eviden-

4. J. Fourquet - Les mutations consonantiques du germanique. París, 1956, 4. 


\section{LEY DE VERNER Y LENGUAS ROMANCES}

ciar un factor de diferenciación; los acentos caían en las mismas sílabas. Desde los primeros siglos de nuestra era, el latín hablado había confundido la $a$ breve de pater y la $a$ larga de frater. El germánico había confundido la $a$ y la $o$ breves en $a$, la $a$ y la $o$ largas en $o$, lo que explica la diferencia de las vocales tónicas, pero no resuelve el problema.

Hubo que esperar medio siglo antes de que la solución fuese traída por un lingüista dinamarqués Karl Verner (1846-1896) ${ }^{5}$. No se había de parar mientes en las lenguas que fijaban mecánicamente la posición del acento en la palabra, sino en las que guardaban en su sitio primitivo el tono indo-europeo. Ahora bien, el sánscrito presentaba pitá pero bhráta; el griego, patér pero fráter. La anomalía se producía en la palabra donde la consonante interesada se encontraba en posición pretónica: tal era era el criterio diferencial buscado.

Antoine Meillet dio a la ley de Verner una formulación particularmente clara y precisa": "La sifflante $s$ et les spirantes $f, t h, k h, k h w$ sont devenues sonores entre deux éléments sonores, dont l'un est l'élément vocalique de la première syllabe du mot, quand le ton hérité de l'indo-européen, tombant sur cette syllabe, n'en empêchait pas la sonorisation".

Quisiéramos enseñar que la ley de Verner no interesa sólo las lenguas germánicas con fecha anterior a nuestra era, sino también la evolución mucho más tardía de las lenguas romances.

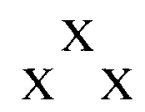

Algunas observaciones preliminares se imponen.

En primer lugar, conviene ser muy prudente en el uso, como ejemplos, de las formas verbales, pues a veces intervienen hechos de analogía entre formas fuertes y formas débiles.

En segundo lugar, la actuación de la ley de Verner es mucho más delicada en itálico que en germánico. Puede, por supuesto, afectar sibilantes y chicheantes, resultados de un mismo grupo del romance común con variaciones de tratamiento a veces dialectales (por ejemplo, sibilantes favorecidas por la Italia del Norte, y chicheantes por la Italia peninsular). Hay grupos que tienen una determinación asegurada de sonora o de sorda: así el grupo dy siempre dará una sonora (podiu/poggio, mediu/mezzo, -idiare/-eggiare; el grupo $k y$, siempre una sorda (brachiu/braccio, glacia/ghiaccia, aciariu/ acciaio). Luego hay que buscar grupos romances que podríamos llamar

5. K. Verner - Zeitschrift für vergleichende Sprachforschung auf dem Gebiete der indogermanischen Sprachen begr. v. A. Kuhn. Göttingen, 1877, XXIII.

6. A. Meillet - Caractères généraux des langues germaniques. París, 1949, 48. 


\section{ENRIQUE GUITER}

"sensibles", los que no tienen una vocación de sonorización o de ensordecimiento tan acusada que la ley de Verner no pueda funcionar.

Sin duda el italiano es el en que la ley de Verner tuvo más importancia; sin embargo, su papel siempre no fue distinguido claramente.

J. Nicolas ${ }^{7}$ ve influencias extrañas en las diferencias de tratamiento: " $t s$ issu de $t+y o d$ n'a pas varié: uitium/uetyu/vetsu/vezzo (phon. véttso). Mais sous l'influence de nombreux mots français, cette évolution phonétique est assez souvent contrariée, et l'on a dans un certain nombre de cas $t+$ yod $\rightarrow d j$ : rationem/radjóne (orthographié ragione)... $s+$ yod $\rightarrow$ tx: basium/ basyu/bacio (phon. bátxo). Mais dans un certain nombre de cas (mots empruntés au français ou aux dialectes du Nord de l'Italie) $s+$ yod $\rightarrow d j$ : sur casus "chute, accident" casionem/cagione (phon. cadjóne)". Las influencias invocadas no parecen pertinentes, ya que tanto el francés como los dialectos italianos del norte corresponden con sibilantes a las chicheantes italianas.

R. Menéndez Pidal $^{8}$ no hace distinción entre las varias evoluciones italianas: "Ambos grupos (ty y $c y$ ) se habían asibilado en el latín vulgar, y el italiano los distingue bien con dos sonidos sordos: $t y \rightarrow z z$ (variedad sorda de $z z$ cuasi lts y $c y \rightarrow c c i$ (esto es $c h$ ); de una parte titione/tizzone, uitiu/vezzo, tristitia/tristezza, puteu/pozzo, acutiare (de acutus)/aguzzare; y de otra parte aciariu/acciaio, corticea/corteccia...". Notemos que tizzone no aparece en italiano sino después del siglo $\mathrm{XIV}^{9}$ y que Dante emplea una forma fuerte tizzo que viene del caso sujeto; aguzzare es una forma verbal débil: en ambos casos puede haber influencia analógica de una forma fuerte.

E. Bourciez ${ }^{10}$ hace intervenir la posición del acento tónico: "Le ty parait avoir été assibilé le premier, puisqu'il l'était déjà au III ${ }^{\mathrm{e}}$ siècle, et a abouti partout à ts (puteum, it. pozzo)... Quant à l'Italie Centrale, elle n'offre la sonore dj que devant l'accent (it. ragione/rationem)."

Y más lejos: "La sourde $t s$ représente en toscan ty latin derrière l'accent, pozzo/puteum, prezzo, giustezza... La sourdè $t x$ représente sy derrière l'accent (bacio/basium, cacio/caseum)... La sonore $d j$ répond à ty devant l'accent (pregiare, ragione/rationem) et à sy devant l'accent (prigione, cagione/ occasionem)". Esta influencia de la posición del acento ya había sido entendida por W. Meyer-Lübke ${ }^{11}$, pero, en el caso del italiano, no se explica tan claramente como E. Bourciez.

7. J. Nicolas - Cours de philologie italienne. Nice, 1972, 38 et 39.

8. R. Menéndez Pidal - Manual de gramática histórica española. Madrid, 1949, 149.

9. C. Battisti y G. Alessio - Dizionario Etimologico Italiano. Firenze, 1957, V, 3806.

10. E. Bourciez - Eléments de linguistique romane. París, 1967, 170-490.

11. W. Meyer-Lübke - Grammaire des Langues Romanes. París, 1900, I, 456. 


\section{LEY DE VERNER Y LENGUAS ROMANCES}

Si agrupamos los hechos italianos tales como aparecen por ty: pláteal piazza, pétia/pezza, tristítia/tristezza, púteu/pozzo, uítiu/vezzo, palátiu/ palazzo, mátea/mazza,...

ratióne/ragione, statióne/stagione, captiatióne/cacciagione, pretiáre/pregiare, indutiáre/indugiare,...

y por $s y$ :

básiu/bacio, cáseu/cacio, camísia/camicia, consuo/cósio/cucio,... (oc)casióne/cagione, pre(hen)sióne/prigione, ma(n)sióne/magione, pe(n)sióne/pigione, pi(n)siáre/pigiare, phaseólu/fagiuolo,...

y aunque muchos abstractos en -one han venido por vía culta, todavía tenemos efectivos suficientes, venidos por vía popular, para evidenciar la oposición entre un grado fuerte sordo después del acento y un grado débil sonoro antes del acento.

\section{$\mathrm{X}$ \\ $\mathrm{X} X$}

Por varias razones, los hechos no resultan tan bien documentados en las lenguas romances occidentales: las vocales finales, a excepción de $a$, cayeron regularmente en las lenguas de Galia y el catalán; el español propendió a neutralizar la oposición sorda/sonora, tendencia que prevaleció definitivamente en provecho de la sorda a principios del siglo XVI.

Sin embargo, ejemplos bastante claros se encuentran en estas lenguas, y se transparentan en el español medieval gracias a las grafías diferentes, $c$ y $z$, de la sorda y la sonora ${ }^{12}$ en los herederos de la ty del romance.

A partir de unas palabras romances con ty postónica: capítia, cupidítia, mátea, palátiu, pétia, pigrítia, plátea, prétiu, tristítia,... o con ty pretónica: pretiáre, ratióne, satióne, titióne, uenatióne, uitiáre,... encontramos:

el francés place, pièce, paresse, y raison, saison, venaison, priser,...

el provenza $1^{13}$ plaço, peço, tristesso, cabesso, ... y resoun, sesoun, tisoun, presa,...

el catalán plaça, peça, maça, cabeça,... y raó, saó, tió, prear,...

el español medieval plaça, pieça, prieço, maça, cabeça,... y razón, sazón, tizón, vezar,...

el portugués praça, peça, paço, preço, cobiça,... y razão, sazão, prezar, vezar...

12. R. Pellen - "Poema de Mio Cid", Dictionnaire lemmatisé des formes et des références. París, 1979. J. Corominas - Diccionario crítico etimológico de la lengua castellana. Berna, 1954.

13. F. Mistral - Tresor dóu Felibrige. Aix, 1879-86. 


\section{ENRIQUE GUITER}

Meyer-Lübke asocia estos hechos con los hechos italianos, y atribuye a la situación del acento la alternación sorda/sonora (sonora reducida a zero en catalán).

Parece extraño que romanistas posteriores hayan buscado otras causas a la presencia de la sorda, sea la analogía, sea una geminación de la $t$.

E. Bourciez ${ }^{14}$ cita el ejemplo "celt. pettia, pièce" (p. 69) e indica (p. 155): "Le mot place remonte à plattea (cl. platea, sans doute influencé par un adjectif plattus d'où vient le fr.. plat). Quant au suffixe -itia, qui devient -ece, -esse (dans mollesse, paresse, etc). il avait dû se changer en -icia". Añade también en los Eléments ${ }^{10}$ : "En Ibérie on a eu aussi $d z$, et les mots où l'a. espagnol présente $t s$ s'expliquent par un groupe tty originaire".

R. Menéndez Pidal ${ }^{8}$ propone la misma explicación: "Las confusiones entre la sonora $z$ y la sorda $c ̧$ son muchas: arcione (diminut. de arcus) arçón, pero más comúnmente se escribía en lo ant. arzón; vice versa: platea plaça; matea maça; potione poçon, poçoña, ponçoña, menos frecuente pozoña; capitiu cabeço cabeça... La causa de hallarse $c$ puede ser el cultismo que detuvo la sonorización, cultismo manifiesto en plaça, por mantener $p l$-. Otras veces la causa será una duplicación de la consonante oclusiva por efecto de la yod".

No se puede negar que una influencia exterior haya actuado en el caso de platea, no forzosamente la influencia del latín: la conservación de la $a$ en francés es tan anormal como la de $p l$ en español. Se pensaría en una lengua vecina, catalán o aragonés para el español, provenzal para el francés.

Nos limitamos estrictamente a ejemplos en los cuales la posición postónica determina la existencia de un grado fuerte sordo, según la misma expresión de la ley de Verner; y prescindimos de accidentes de misma causa en los cuales el grado fuerte resulta del mantenimiento de una sonora, v.g. plága/llaga pero regále/real. Sin embargo, el conjunto de hechos invocados y su acuerdo en varias lenguas romances no permiten que dudemos.

Meyer-Lübke tenía toda la razón cuando escribía ${ }^{11}$ a propósito de ty romance: "L'influence de l'accent est beaucoup plus grande ici que dans n'importe quel cas... Ty posttonique devient déjà en latin vulgaire ts redoublé, lequel persiste en italien, est simplifié ailleurs en simple ts... Ty protonique suit une tout autre voie. Il était déjà devenu en latin vulgaire $t s i$, puis ce $t$ si protonique est devenu sonore de même que les explosives sourdes...".

Reducida, es verdad, a un solo fonema, cuando se quiere abarcar más de una lengua romance, la formulación de Meyer-Lübke no deja de recordar la de la ley de Verner, que valía para el conjunto de las fricativas del germánico común. Es un rasgo más, que acerca itálico y germánico. En germánico 


\section{LEY DE VERNER Y LENGUAS ROMANCES}

el accidente tiene que ser antiguo, ya que el tono indo-europeo todavía desempeñaba un papel; en itálico no data más que de la época romance primitiva, y el tono latino es entonces el factor determinante. 
